\title{
Diagrama tangente: útil recurso do programa Ester 2.1 para projeção estereográfica em Geologia
}

\author{
Celso Dal Ré Carneiro ${ }^{1}$, Giovanni Amaral Guimarães², João Paulo da Ponte Souza ${ }^{3}$, Gisele Francelino Miguel ${ }^{4}$ \\ 1- Programa de Pós-Graduação em Ensino e História de Clências da Terra, Instituto de Geociências, Universidade Estadual de Campinas, Campinas, SP. Bolsista do \\ CNPQ, BRASIL. E-MALL: CEDREC@IGE UNICAMP.BR \\ 2- Graduando em Geologia, Instituto de Geociências, Universidade Estadual de Campinas, Campinas, SP. E-mail: glovanniamaral95@gmall.com \\ 3- Geólogo, Mestrando em Geologia de Petróleo, Centro de Estudos de Petróleo (Cepetro), Universidade Estadual de Campinas, Campinas, SP. E-mall: joao. \\ PONTE.SOUZA@GMAIL.COM \\ 4- Geologa, Mestranda do Programa de Pós-Graduação em Ensino e História de Clências da Terra, Instituto de Geociências, Universidade Estadual de Campinas, \\ Campinas, SP. Bolsista Capes, Brasil. E-mall: giselefmiguel@yahoo.com.br
}

Abstract: Studies and applied works of structural analysis depend on specialized softwares and resources for 3D modeling. This article presents the results of a research that aimed to evaluate the effectiveness of version 2.1 of the Ester stereographic projection system for Structural Geology.-The evaluation of the software involved tests with structural data of different geological contexts, submitted to graphic treatment in packages such as Stereonet, Stereonet9, QuickPlot, Dips, OpenStereo and other softwares accessible at low cost. The study privileged the truly free (open) softwares. One advance was the inclusion of the Tangent Polar Diagram into the Ester package. The tool helps to differentiate, in folded regions, conical folds from cylindrical folds. In the comparative evaluation, it was observed that diagrams produced by Ester 2.1 are similar to those generated by other progra$\mathrm{ms}$. The advantage relates to resources that are not available in the other softwares analyzed. It is concluded that, in addition to resources heretofore unavailable, the tests showed good functionality. The experience signals new directions in the search for educational alternatives for teaching-learning in Structural Geology.
Manuscrito:

Recebido: 29-Set-17

Corrigido: 24-0ut-17

Aceito: 14-Fev-18

Citation: Carneiro C.D.R., Guimarães G.A, Souza J.P.P., Miguel G.F. 2018. Diagrama tangente: útil recurso do programa Ester 2.1 para projeção estereográfica em Geologia Terræ Didatica, 14(1):15-26. URL: http://www.ige.unicamp. br/terraedidatica/

Keywords: Structural Geology, teaching-learning, geological structures, stereographic projection software, Windows.

\section{Introdução}

A Geologia é uma ciência interpretativa e histórica (Frodeman 2010) que requer do geocientista um conjunto de habilidades de pensamento e investigação pouco comuns em outras ciências. Para os geocientistas, e em todas as especialidades das Geociências, "a mente humana é indiscutivelmente a ferramenta mais importante" (Kastens et al. 2009). A capacidade de pensamento tridimensional de alto nível é crítica para os geólogos e constitui atributo distintivo dos profissionais dedicados às Ciências da Terra (King 2008). Railsback (2002a, 2002b) ilustra o tipo de raciocínio espaço-temporal dos geólogos por meio de duas representações de relevo e algumas interpretações que podem ser feitas a partir da "leitura geológica" da paisagem (Carneiro \& Carvalho 2012, Fig.1). A visualização é, portanto, um aspecto da habilidade espacial que precisa ser desenvolvida no processo de ensino-aprendizagem.

A projeção estereográfica cumpre, por vários motivos, papel decisivo na aprendizagem significativa em Geologia Estrutural e no desenvolvimento de visão espacial pelo estudante. Carneiro \& Carvalho (2012) salientam ser comum, nas disciplinas de Geologia Estrutural, que muitos alunos obtenham resultados insatisfatórios nas avaliações de aprendizado. Isso requer maior esforço dos professores e alunos em busca de novas alternativas educacionais. Desde 2006, um dos autores (CDRC) orienta pesquisas de estudantes de graduação, buscando: (a) atualizar programas de projeção estereográfica para Geologia em ambiente Windows, (b) pesquisar inovações em visualização 3D e (c) investigar aspectos inconfundíveis da aprendizagem significativa de Geologia Estrutural. 
Este artigo apresenta os resultados de pesquisa que objetivou avaliar a eficácia da versão, que neste artigo denominamos Ester 2.1, para projeção estereográfica em Geologia Estrutural, por meio da comparação com diagramas gerados em outros programas livres ou acessíveis a baixo custo pela Internet. O sistema Ester havia sido lançado em 1996, em obra co-editada por Unicamp-CPRM-IPT (Carneiro 1996), mas ficou ultrapassada, em vista da evolução dos sistemas operacionais. Um recurso novo oferecido pela versão 2.1 é o Diagrama Tangente (Groshong Jr. 2006), cujas características são brevemente descritas nesta comunicação. Os testes envolveram dados estruturais de diferentes contextos geológicos, que foram objeto de tratamento gráfico em pacotes como Stereonet, Stereonet9, QuickPlot, Dips, OpenStereo e outros programas acessíveis a baixo custo. Privilegiaram-se os que são realmente abertos, como Stereonet, Stereonet9 e OpenStereo.

\section{Ferramentas digitais para projeção estereográfica}

A projeção estereográfica é essencial em Geologia, porque permite solucionar problemas geométricos tridimensionais, por meio da representação de feições espaciais em um plano. As aplicações práticas são incontáveis, inexistindo limites de escala ou de posição espacial para os objetos analisáveis. Os diagramas resultantes podem ser produzidos manualmente, sendo muito úteis no ensino-aprendizagem de Cristalografia, Geologia Estrutural, Sismologia e Paleomagnetismo. Como a projeção manual é lenta e trabalhosa, o auxílio de computadores pessoais favorece a produção expedita de diagramas de projeção estereográfica, requerendo, contudo, softwares que realizem as funções requeridas. Sistemas que geram redes estereográficas digitais participam de ampla variedade de atividades humanas que dependem de visualização de estruturas geológicas, como exploração e explotação de água subterrânea, planejamento regional, estudos tectônicos, estudos de implantação de obras civis e fundações, estudos estruturais de escalas local a regional, mapeamentos geológicos, estruturais e geomorfológicos etc.

Muitos aplicativos atuais de modelagem tridimensional de estruturas geológicas, sistemas de gerenciamento e programas comerciais de modelagem atingiram avançado estágio de desenvolvimento, mas a maioria destina-se às áreas de petróleo e mineração, para tratar dados de perfuração e perfis sísmicos. $\mathrm{O}$ alto custo de aquisição de licenças se coaduna com o elevado investimento financeiro que caracteriza as áreas de exploração mineral de petróleo. Há aplicações similares, disponíveis em ambiente CAD (Jacobson 2001a, 2001b), menos potentes e menos onerosas, porém ainda restritas, sobretudo se pensarmos no ambiente educacional (Carneiro \& Carvalho 2007, 2012).

A projeção estereográfica pertence à categoria de aplicativos de custo relativamente baixo, existindo programas de acesso livre: Stereonet (Roeller \& Trepmann 2003-2008), Stereonet9 (Allmendinger 2017), Dips, GEOrient 9.1 (Holcombe 2003), QuickPlot, OpenStereo e outros. OpenStereo é um programa nacional para projeção estereográfica e análise de dados estruturais (Grohmann \& Campanha 2010, Grohmann et al. 2011). O sistema de projeção estereográfica para Geologia Estrutural Ester reune rotinas para análise estatística e visualização 3D, em ambiente Windows, e também atende ao conceito de Software Livre:

\section{Apesar de muitos programas livres serem gra- tuitos, "Livre" (Free) está ligado ao conceito de Liberdade. O conceito diz respeito à liberdade dos usuários de executar, distribuir, estudar, modificar e melhorar o programa. Para que um programa satisfaça essas condições, é necessário que o código-fonte esteja disponível ao usuário, independente do programa ser distribuído gra- tuitamente via Internet ou vendido comercial- mente (Free Software Foundation 2000).}

A tecnologia computacional favorece a coleta, tratamento e interpretação de grandes volumes de dados. Inúmeros estudos de aplicação da análise estrutural e modelagem tridimensional dependem do uso de computadores, softwares e recursos especializados, cujo domínio deve ser estimulado desde o curso de graduação, pois o computador continuará a ser cada vez mais utilizado no ensino de Geologia Estrutural, tanto no campo como em sala de aula (Burger et al. 2004).

Blenkinsop (1999) analisa dificuldades comuns aos alunos que estudam projeção estereográfica, concluindo que há três tipos de problemas: (a) baixa familiaridade com o uso de bússola e com a notação correta de dados de orientação de planos e retas; (b) deficiências de visualização espacial que impedem a compreensão de princípios e práticas de projeção, embora os alunos possam, às vezes, executar bem determinados exercícios, sem dispor desse entendimento; (c) falta de interesse ou des- 
conhecimento da importância e utilidade da técnica. Os materiais eletrônicos de aprendizagem são altamente vantajosos para a "aprendizagem, quando usados em conjunto com métodos tradicionais de ensino" (James \& Clark 1996). Desse modo, ponderamos que o desenvolvimento da habilidade de visualização $3 \mathrm{D}$ pelo estudante deva preceder a plena utilização de tais recursos.

\section{Breve histórico}

$\mathrm{Na}$ segunda metade do século XX surgiram softwares de projeção estereográfica, que até o início dos anos 1980 se restringiam a computadores de grande porte. Nessa época disputavam espaço os sistemas dedicados (mainframes) e o universo de microcomputadores pessoais que então engatinhava. Em fins dos anos 1980 foram desenvolvidos softwares para microcomputadores (Gurth 1987; Stowe 1988, dentre outros), sistemas complementares a ambiente GIS (Knox-Robinson \& Gardoll 1998) e programas shareware, como QuickPlot (Van Everdingen \& Van Gool 1990; Van Everdingen et al. 1992). Nas últimas duas décadas apareceram inúmeras aplicações, que não se restringem ao ambiente acadêmico.

O Instituto de Pesquisas Tecnológicas do Estado de São Paulo (IPT) desenvolveu programas em língua portuguesa e de tecnologia nacional para projeção estereográfica de dados de estruturas geológicas em ambiente MS-DOS, que ofereciam autonomia e minimizavam a dependência do geólogo em relação a softwares estrangeiros. As pesquisas culminaram nas versões para microcomputador dos programas ESTER e TRADE (Carneiro et al. 1996; Carneiro 1996). A publicação possibilitou divulgar os programas, que hoje estão desatualizados. Surgiram outros recursos para modelagem e representação tridimensional, mas permanecem inéditas algumas funcionalidades de análise de dados estruturais contidas em Ester 1.0 e Trade, como a determinação de direções principais de deformação.

Desde 2006, sucessivos projetos de pesquisa coordenados pelo autor CDRC foram realizados na Unicamp com apoio do Programa PIBIC/ $\mathrm{CNPq}$, buscando-se atualizar programas integrados e desenvolver sistemas de visualização 3D de estruturas geológicas (Mizuno \& Carneiro 2007, 2008; Carneiro \& Carvalho 2012; Ponte \& Carneiro 2013). Após sucessivas pesquisas, o objetivo de expandir as funcionalidades dos programas foi atingido, chegando-se a uma versão experimental compatível com Windows 10. Utilizando linguagens de programação modernas, foram desenvolvidas novas interfaces gráficas. As rotinas de contagem estatística e de exibição de dados oferecem ao intérprete a possibilidade de alterar os formatos de saída de diagramas e escolher padrões de apresentação mais adequados. A entrada de dados permite fazer acréscimos e correções; reconfigurou-se o processo de produção de diagramas de saída, de tal modo que os resultados podem ser salvos em arquivos de imagens, para uso como ilustrações, sem necessidade do programa original para abrí-los.

\section{Objetivos}

Os objetivos centrais deste artigo compreendem validação e aprimoramento de sistema de projeção estereográfica robusto para tratamento de dados geológico-estruturais em sistema operacional Windows. Descrevemos os resultados da avaliação crítica da versão Ester 2.1, com base em testes comparativos que buscaram avaliar sua eficácia e eficiência.

Desde que foi publicada a primeira versão de Ester e Trade (Campanha et al. 1996), em ambiente DOS (Disk Operating System), surgiram inovações, com destaque para a rede de projeção denominada Diagrama Tangente, que já compõe a versão analisada. A versão 2.1 do programa Ester realiza a representação ciclográfica ou polar de dados de feições planares e lineares; elabora diagramas de roseta e diagramas tangente (ver descrição adiante); desenvolve testes estatísticos e de análise de falhas, além de possibilitar a determinação das orientações principais de encurtamento e estiramento tectônico (Souza \& Carneiro 2013). Uma vez superada a etapa de desenvolvimento, Ester 2.1 deveria ser objeto de testes antes de ser publicado; dessa forma, realizamos avaliação prática e testamos a confiabilidade e precisão para usos técnico-científico, educacional e profissional. Os resultados são apresentados e discutidos neste artigo.

\section{Materiais e métodos}

Adotou-se um caminho metodológico para validação do programa Ester e dos aplicativos a ele vinculados que envolve recuperação de dados da literatura e obtenção de dados originais de campo. A parte prática da pesquisa envolveu inserção 
e tratamento de dados estruturais de diferentes procedências.

O estudo baseia-se na versão mais recente disponível de Ester 2.1. Os critérios utilizados na pesquisa precisam ser rigorosos, porque são uma etapa de validação de Ester que opera em ambiente Windows 10 . O método de trabalho envolveu a produção de diagramas em softwares distintos, a partir de idênticos conjuntos de dados, para facilitar a comparação visual. Os diagramas gerados por Ester 2.1 foram comparados a diagramas feitos com outros softwares, buscando-se identificar possíveis discrepâncias, deficiências ou redundâncias entre os diagramas. Os dados empregados constam das tabelas contidas no artigo. Ao longo do projeto os problemas foram enumerados e investigados, para aprimorar o software e torná-lo harmonioso e amigável.

Os testes de avaliação do software Ester 2.1 consistem em: estudar os recursos que o programa oferece, testar sua eficácia e comparar os diagramas por ele produzidos com pacotes similares existentes no mercado. As etapas envolveram:

1. Avaliação do ambiente computacional.

2. Inserção do recurso de elaboração do Diagrama Tangente no programa Ester.

3. Produção de diagramas de análise estatística mediante inserção e tratamento de dados reais.

4. Exame comparativo dos diagramas da etapa (3) com aqueles originados em outros programas.

\section{Resultados}

\section{Avaliação do ambiente Ester 2.1}

O software Ester 2.1 possui ferramentas que facilitam o trabalho do usuário; a barra de ferramentas superior facilita a inserção de dados (Fig. 1). Os dados precisam ser inseridos em formato Clar, tanto para planos como para retas; indicam-se o azimute do mergulho e o ângulo de mergulho. A barra de ferramentas lateral acompanha a tendência moderna de muitos programas de desenho gráfico, pois oferece algumas opções de representação dos dados analisados: (a) plotagem de diagramas; (b) plota- gem em separado para dados lineares ou planares; (c) plotagem dos dados em ciclogramas ou polos; (d) plotagem dos dados em gráficos de roseta ou (e) gráficos de análise estatística. Mais de uma alternativa pode ser escolhida pelo usuário, o que aumenta a capacidade de visualização tridimensional.

A barra de ferramentas principal do aplicativo permite importar e exportar arquivos de/para os softwares DIPS e Stereonet. Outra ferramenta salva os diagramas gerados em arquivos de imagem como .jpg, .tiff, .bmp e outros. São disponíveis as funções de gerenciamento das janelas que se abrem no próprio ambiente Ester 2.1 (Fig. 1). O comando ${ }^{\wedge} \mathrm{N}$ (Arquivo - Novo) cria uma nova janela, ao se dar início a uma nova sessão do programa. O ambiente Ester permite inserção concomitante de dados, cálculo de ângulo entre estruturas, determinação da atitude resultante de interseção de dados, alteração das formas de controle estatístico dos dados em análise, conversor de medidas e rotação de dados estruturais. Existe ainda a possibilidade de produzir um diagrama tangente a partir de um mesmo conjunto de dados, além de ser possível aplicar os métodos de Arthaud (1969), Aleksandrowski (1985) e Angelier (1979).

Pode ser questionado que a restrição de entrada de dados ao formato Clar pode afastar usuários inexperientes, mas o quesito é compensado pela disponibilidade da ferramenta de conversão. $\mathrm{O}$ ambiente Ester 2.1 conta com barra lateral para visualização dos dados, dando um aspecto moderno e prático ao sistema. Os dados são introduzidos no mesmo ambiente em que são trabalhados, por meio 
da barra de ferramentas superior ou da ferramenta de introdução de dados, em casos com múltiplos dados. Isso permite que o usuário fique imerso no aplicativo durante toda a utilização.

\section{Diagrama Tangente}

O Diagrama Tangente (Fig. 2) objetiva facilitar a interpretação de estruturas geológicas, tendo sido construído com base em princípios estabelecidos por Hubbert (1931), Hitson (1928) e Nettleton (1931). Também conhecido como Diagrama Polar Tangente, foi popularizado por Bengtson (1980).

O método de plotagem utiliza notação Clar, mas o rumo do mergulho do plano a ser plotado é lido na borda do diagrama e a pendente do mergulho aumenta do centro do diagrama para a borda. Cada vetor é representado pela ligação entre o polo e o centro do diagrama. Estruturas com alto ângulo de mergulho são plotadas proporcionalmente mais longe do centro do que mergulhos baixos. Uma escala auxiliar permite estender a faixa de mergulhos até valores próximos a $80^{\circ}$. Acima desse valor o exagero na escala de mergulhos é excessivo e o uso do diagrama deve ser evitado. Apesar de ter sido pouco difundido no meio técnico (Bengtson 1980), o diagrama tangente é útil para estudo de dobras cilíndricas e cônicas (ver adiante).

Bengtson (1980) descreve métodos que otimizam a utilidade do diagrama, assinalando como vantagens do diagrama tangente o fato de ser desnecessário rotacionar a rede para fazer plotagens, ou converter planos em polos e vice-versa: "polos de volta para planos". O autor divide as aplicações em duas categorias: (a) análise de dados e (b) análise de dobras.

\section{Análise de dados}

O Diagrama Tangente favorece o estudo de relações entre mergulhos reais (verdadeiros) e mergulhos aparentes, em diferentes situações:

(a-I) Determinar um mergulho aparente de um plano a partir do verdadeiro.

(a-II) Determinar o mergulho real de um plano a partir de dois mergulhos aparentes.

(a-III) Determinar a linha de interseção de dois planos. A determinação baseia-se no princípio de que, se dois planos se interseptam, eles possuem mergulhos aparentes iguais no plano vertical que contém a linha de interseção.

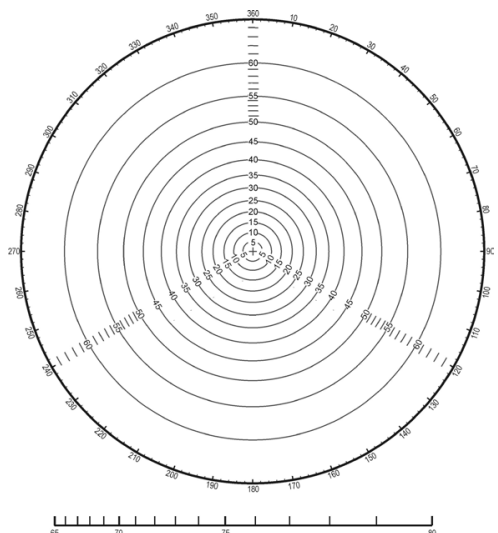

Figura 2. Diagrama Tangente para tratamento de dados estruturais

\section{Análise de dobras}

Tradicionalmente, o estudo da geometria de dobras divide-as para efeito de classificação em dois grandes grupos: dobras cilíndricas e cônicas. Nas redes estereográficas, os polos da superfície dobrada em dobras cilíndricas distribuem-se segundo uma guirlanda que acompanha o traçado de um círculo máximo, enquanto nas dobras cônicas o arranjo assume o formato aproximado de um círculo mínimo. O diagrama tangente introduz alguma exatidão adicional para fazer a discriminação entre os dois tipos, desde que os dados tenham sido obtidos para apenas um caimento de dobra.

(b-I) No diagrama tangente, dobras cilíndricas apresentam distribuição em formato de reta quando são plotados os polos da superfície dobrada (Bengtson 1980); o vetor gerado entre a reta perpendicular aos dados da dobra e o centro do diagrama indica o vetor referente à linha de crista (eixo b), que é uma reta cuja translação no espaço determina a forma da dobra cilíndrica. Para uma dobra cilíndrica horizontal, a linha reta dos dados passa pelo centro do diagrama; caso exista algum ângulo de caimento, o mergulho do vetor indica o caimento da dobra.

(b-II) Dobras cônicas apresentam um ponto de fechamento da dobra, denominado vértice; são divididas nos tipos I e II. No diagrama tangente, a plotagem dos dados de mergulho da superfície dobrada apresenta formato de hipérbole, ao contrário da linha reta obtida no caso das dobras cilíndricas. Um anticlinal cônico do tipo I possui o vértice caimento-acima (up-plunge), enquanto um anticlinal cônico do tipo II possui o vértice caimento-abaixo (downplunge) (Bengtson 1980). A posição da 
concavidade da hipérbole depende da posição do vértice: nas dobras cônicas do tipo I com relação ao caimento da dobra a concavidade da linha gerada pelos dados estruturais apontará para o centro do diagrama (nesse caso temos um anticlinal cônico up-plunge ou sinclinal cônico downplunge). $\mathrm{Na}$ situação inversa (dobras cônicas tipo II) a concavidade apontará para as bordas do diagrama (anticlinal cônico downplunge e sinclinal cônico up-plunge). Nesse tipo de dobra é possível visualizar a linha de crista, vetor que reflete o caimento da dobra, por meio da ligação do centro do diagrama com o centro da hipérbole.

A análise dos dados plotados deve ser realizada com cautela, tendo em vista que o alvo são estruturas de maior escala. Portanto, dados discordantes devem ser registrados em menor quantidade sugerindo a presença de estruturas menores, secundárias, com relação à estrutura pesquisada.

Mynatt et al. (2007) expandem a classificação de dobras cilíndricas e cônicas para acomodar uma terceira categoria, a sela estrutural, porém ainda não estão disponíveis exemplos de tratamento desse tipo de dobra em diagrama tangente.

\section{Diagramas de análise estatística}

Para esse teste foi inserido, no Software Ester 2.1, um grupo de dados de 90 medidas planares (Tab. 1), o resultado gerado pelo programa acha-se na Figura 3. Cada dado individual pode ter sua cor alterada a fim de obter melhor visualização. A imagem gerada apresenta as seguintes referências: pontos cardeais; tipo de projeção utilizada; o símbolo $\Omega$ invertido indica que a projeção utiliza o hemisfério inferior. Assinala-se ainda o tipo de diagrama em que os dados estão dispostos: diagrama de Schmidt ou Schmidt-Lambert.

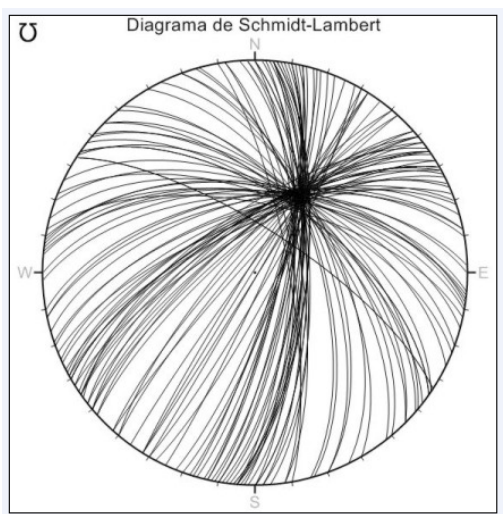

Figura 3. Diagrama resultante do tratamento de dados no software Ester 2.1

O programa permite dispor ao mesmo tempo mais de um tipo de diagrama (polar e ciclográfico) o que facilita a interpretação dos dados. Outra opção disponível no software Ester 2.1 é a de geração de gráficos em roseta, por uma ferramenta interna do Software e um menu de fácil utilização (fig. 4). Os gráficos em roseta ilustram as direções das estruturas representadas.

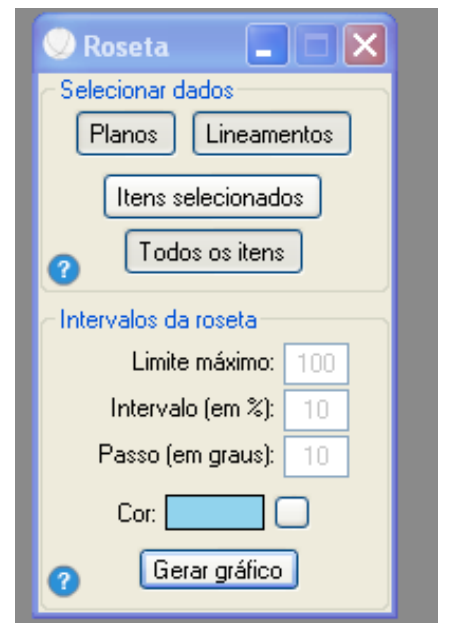

Figura 4. Menu de opções para geração do gráfico em roseta

Tabela 1. Medidas estruturais. Fonte: Furumoto et al. (1996, p.50)

\begin{tabular}{c|c|c|c|c|c|c|c|c|c}
\hline \multicolumn{10}{c}{ Plano ou Linha (Atitude Clar) } \\
\hline \hline $110 / 80$ & $103 / 80$ & $95 / 73$ & $64 / 58$ & $348 / 62$ & $86 / 70$ & $341 / 66$ & $15 / 57$ & $90 / 75$ & $100 / 76$ \\
\hline $108 / 81$ & $330 / 70$ & $96 / 70$ & $74 / 64$ & $350 / 60$ & $88 / 69$ & $340 / 65$ & $98 / 74$ & $324 / 67$ & $330 / 70$ \\
\hline $106 / 80$ & $331 / 72$ & $97 / 72$ & $75 / 65$ & $118 / 82$ & $92 / 72$ & $318 / 78$ & $93 / 75$ & $328 / 75$ & $6 / 60$ \\
\hline $107 / 82$ & $302 / 89$ & $99 / 74$ & $120 / 89$ & $120 / 85$ & $90 / 70$ & $331 / 68$ & $115 / 86$ & $344 / 62$ & $7 / 58$ \\
\hline $100 / 77$ & $302 / 87$ & $360 / 58$ & $122 / 89$ & $310 / 83$ & $322 / 75$ & $335 / 69$ & $322 / 71$ & $333 / 72$ & $8 / 55$ \\
\hline $101 / 78$ & $312 / 81$ & $358 / 60$ & $35 / 60$ & $312 / 85$ & $320 / 75$ & $26 / 56$ & $88 / 72$ & $82 / 75$ & $9 / 56$ \\
\hline $90 / 70$ & $310 / 79$ & $10 / 59$ & $38 / 60$ & $318 / 75$ & $326 / 70$ & $45 / 57$ & $320 / 72$ & $96 / 70$ & $95 / 76$ \\
\hline $94 / 72$ & $40 / 56$ & $12 / 63$ & $34 / 56$ & $320 / 77$ & $325 / 72$ & $70 / 62$ & $332 / 68$ & $95 / 72$ & $330 / 70$ \\
\hline $102 / 77$ & $41 / 58$ & $336 / 67$ & $35 / 60$ & $325 / 74$ & $20 / 56$ & $306 / 87$ & $5 / 58$ & $348 / 60$ & $45 / 60$ \\
\hline $104 / 80$ & $80 / 77$ & $338 / 70$ & $50 / 58$ & $325 / 70$ & $22 / 60$ & $328 / 69$ & $95 / 70$ & $112 / 81$ & $70 / 60$ \\
\hline $103 / 81$ & $82 / 72$ & $60 / 60$ & $52 / 60$ & & & & & & \\
\hline
\end{tabular}

\begin{tabular}{|c|c|c|c|c|c|}
\hline (C) Terrae Didat. & Campinas, SP & v.14 & n.1 & p. $15-26$ & jan./mar. 2018 \\
\hline
\end{tabular}


No Software Ester 2.1 é possível inserir concomitantemente dados planares e lineares em um único arquivo, sendo possível alterar cores em separado para dados isolados. Para ilustrar, foi acrescentado o conjunto de dados da Tabela 2 ao conjunto do exemplo anterior, e que resultou no diagrama da Figura 5. O resultado continua a seguir os padrões estéticos e a simbologia dos diagramas anteriores, enquanto as linhas foram plotadas como pontos no diagrama.

Ferramenta útil que pode ser facilmente acessada pelo programa Ester 2.1 é a de ilustração esta-

Tabela 2. Medidas de atitudes de falhas. Fonte: Torquato et al. (1996, p.111)

\begin{tabular}{|c|c|c|c}
\hline Planos de falhas & Slickensides & Planos de falhas & Slickensides \\
\hline 1) N50E 50SE & $204 / 27$ & 5) N50E 80SE & $223 / 30$ \\
\hline 2) N50E 70SE & $217 / 30$ & 6) N50E 20SE & $170 / 16$ \\
\hline 3) N50E 24SE & $175 / 20$ & 7) N50E 40SE & $190 / 27$ \\
\hline 4) N50E 40NW & $264 / 26$ & 8) N50E 70NW & $243 / 33$ \\
\hline
\end{tabular}

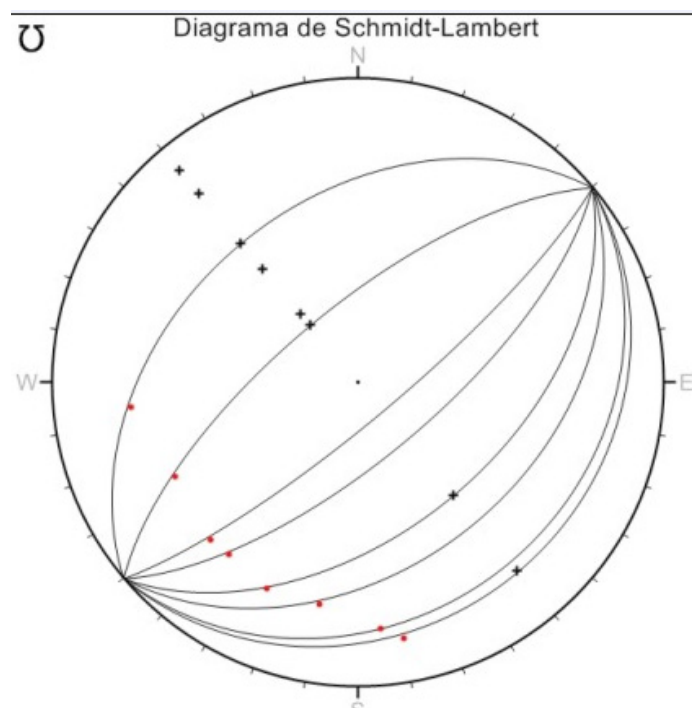

Figura 5. Diagrama resultante do tratamento dos dados da Tabela 2, no software Ester 2.1

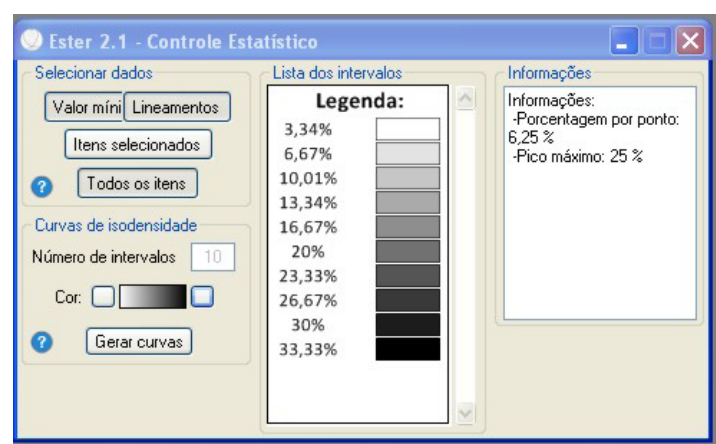

Figura 6. Menu de opções para geração do gráfico de densidade tística de densidade de pontos no estereograma; a função não pode ser exibida concomitantemente às outras funções de geração de diagramas no software. Para exibição do controle estatístico é necessário escolher os dados que serão avaliados, linhas e planos ou apenas um desses, todos os dados ou apenas os selecionados, número de intervalos presentes e a cor que apresentará diversos tons até alcançar a outra cor que também pode ser selecionada. A figura 6 ilustra o menu de Controle Estatístico do programa.

Utilizando os dados da Tabela 3, que reune 15 dados planares e outros 15 lineações, contendo Ester 1.0, é possível elaborar gráfico de densidade de retas, polos e lineamentos (Fig. 7). O diagrama resultante indica a distribuição de curvas na forma de contagens sucessivas.

Tabela 3. Medidas de atitudes de falhas e estrias. Fonte: Torquato et al. (1996, p.112)

\begin{tabular}{c|c}
\hline Planos de falhas & Atitudes das estrias \\
\hline 1) N10E 35SE & $160 / 19$ \\
\hline 2) N30E 50SE & $146 / 32$ \\
\hline 3) N52E 85SE & $224 / 50$ \\
\hline 4) N78E 50NW & $314 / 45$ \\
\hline 5) N69W 30NE & $249 / 33$ \\
\hline 6) N13W 30NE & $150 / 08$ \\
\hline 7) N36W 40NE & $340 / 14$ \\
\hline 8) N58E 80NW & $255 / 52$ \\
\hline 9) N60E vert. & $240 / 40$ \\
\hline 10) N46E 70SE & $204 / 44$ \\
\hline 11) N44E vert. & $224 / 38$ \\
\hline 12) N40E vert. & $206 / 48$ \\
\hline 13) N52E vert & $227 / 50$ \\
\hline 14) N50E 80SE & $216 / 52$ \\
\hline 15) N50E 80NW & $242 / 54$ \\
\hline
\end{tabular}

$\mho \quad$ Diagrama de Schmidt-Lambert

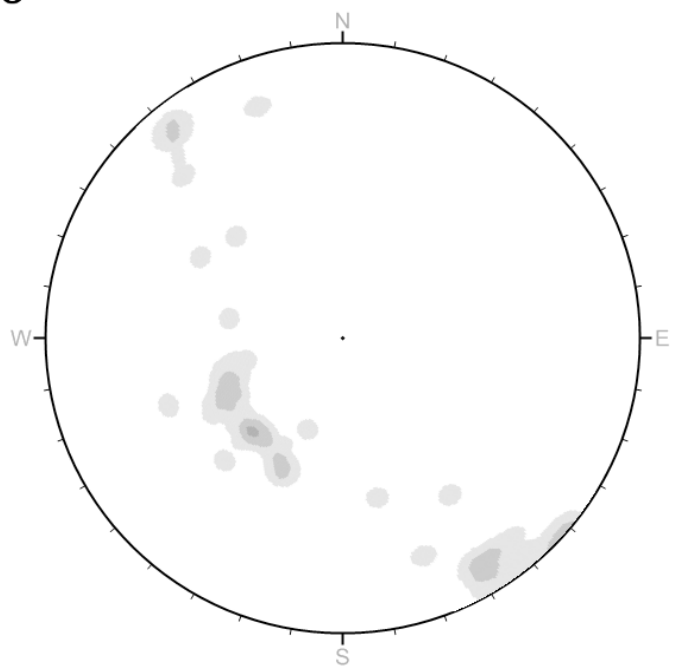

Figura 7. Diagrama resultante dos dados da Tabela 3, com a ferramenta de controle estatístico de Ester 2.1 


\section{Comparação dos diagramas gerados pelos programas}

Com a finalidade de obter comparação direta entre os programas: Ester 2.1, Stereonet9 e OpenStereo, foram gerados diagramas utilizando os dados da tabela 1. O resultado da plotagem dos dados é bastante similar em todos os programas, porém podem ser identificadas algumas imperfeições em todos eles. (Figs. 8 a 12)
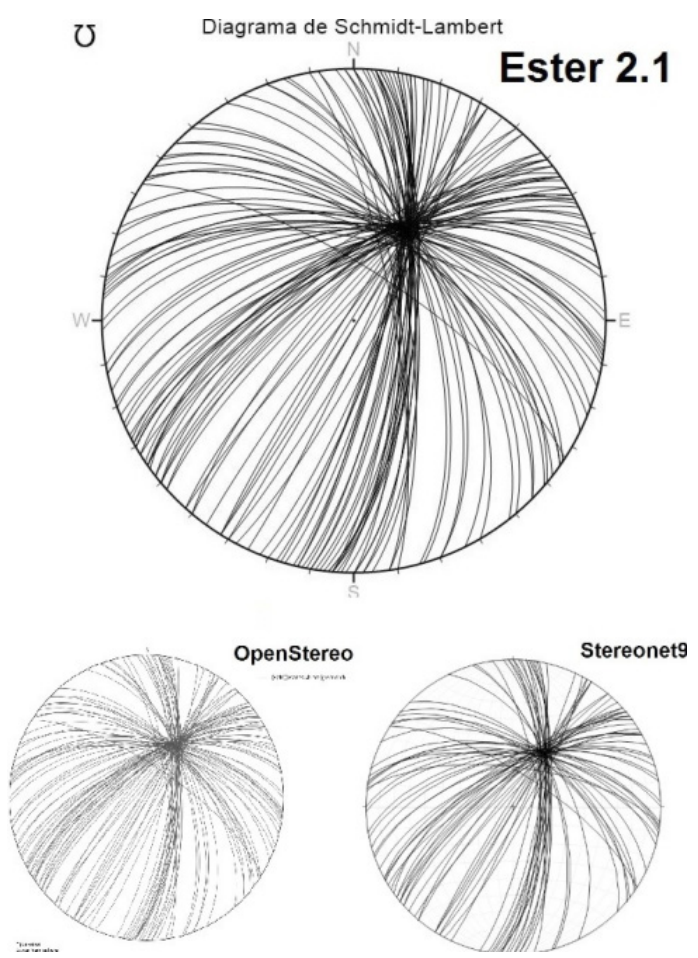

Figura 8. Similaridade de resultados dos softwares Ester 2.1 , Stereonet +9 e OpenStereo, com os dados da Tabela 1

Uma divergência está presente quando se elaboram diagramas de roseta. A fim de ilustrar essa especificidade utilizou-se os dados da Tabela 4 nos três softwares (Ester 2.1, Stereonet9 e OpenStereo). Os resultados estão apresentados na Figura 9. Todos os programas geram um diagrama de roseta a partir da direção de mergulho dos planos; o programa OpenStereo apresenta a opção de gerar o diagrama com dados de direção dos planos. O software Stereonet9 gera o diagrama apenas em $360^{\circ}$, enquanto Ester 2.1 apresenta apenas a opção em $180^{\circ}$. O programa OpenStereo deixa a escolha a cargo do usuário. No Stereonet9 não se pode controlar a porcentagem limite; é sempre de $100 \%$, o que pode dificultar a visualização dos dados; nos outros programas o limite foi ajustado para $30 \%$ a fim de melhorar a resolução da imagem. Outra limitação é o fato de não ser possível retirar a plotagem dos planos no software Stereonet9.

Acerca dos produtos gerados, não há diferença entre os programas Ester 2.1 e Stereonet9. Para inserção de dados planares e lineares em um único diagrama é necessário criar dois arquivos no forma-

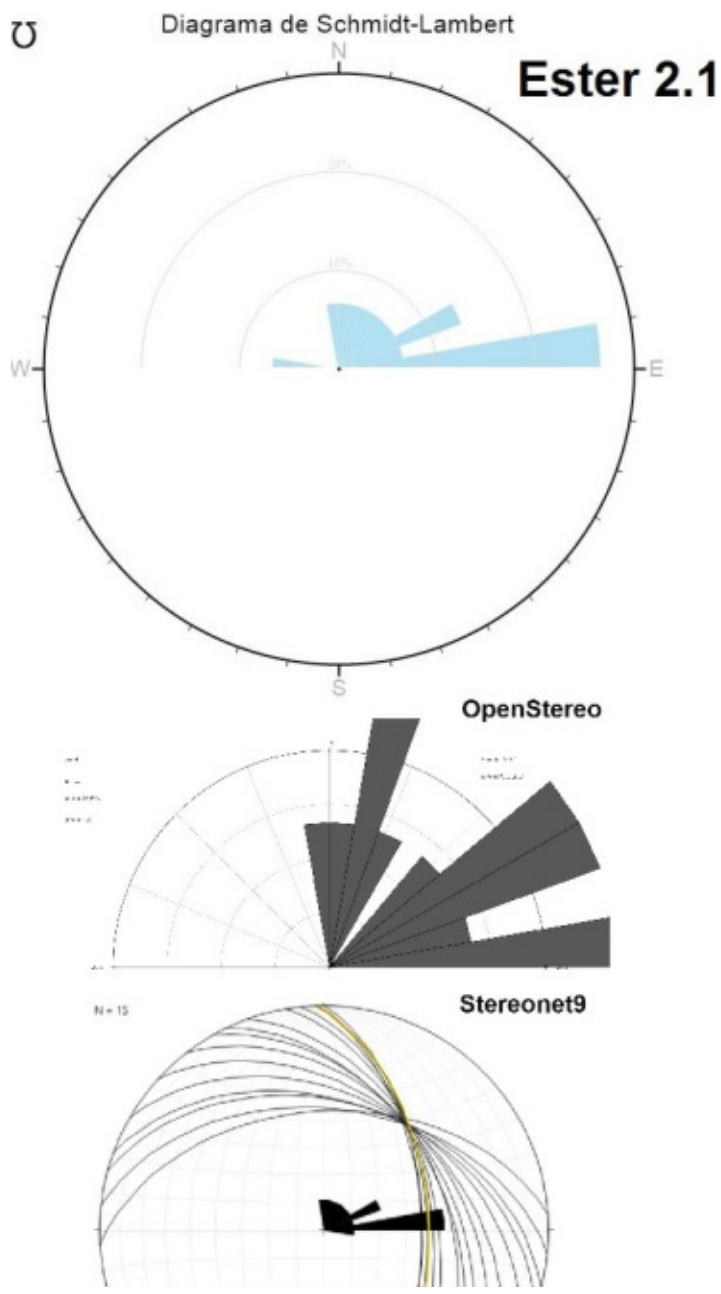

Figura 9. Resultados gerados pelos softwares Ester 2.1, Stereonet9 e OpenStereo, com os dados da Tabela 4. Observam-se diferenças entre os diagramas resultantes; em um deles há uma moda a mais

Tabela 4. Atitudes de uma dobra. Fonte: Torquato et al. (1996, p.79)

Plano ou Linha (Atitude Brunton)

\begin{tabular}{l|c|l|l|l}
\hline 1) N02W 54NE & 4) N60W 40NE & 7) N20W 44NE & 10) N30W 42NE & 13) N70W 42NE \\
\hline 2) N10W 48NE & 5) N26W 44NE & 8) N75W 42NE & 11) N06W 50NE & 14) N48W 40NE \\
\hline 3) N86E 46NW & 6) N-S 54E & 9) N38W 40NE & 12) N84W 45NE & 15) N02W 52NE \\
\hline
\end{tabular}

\begin{tabular}{l|c|c|c|c|c|}
\hline (c) Terrae Didat. & Campinas, SP & v.14 & n.1 & p. 15-26 & jan./mar. 2018 \\
\hline
\end{tabular}


to .txt no caso do software OpenStereo. Exigem-se duas categorias de dados no ambiente do programa Stereonet9 enquanto na versão Ester 2.1 um único conjunto de dados já possibilita a plotagem dos dados. Embora a separação de grupos de dados com relação à direção aconteça de maneira distinta, as porcentagens aparecem de forma visualmente distinta dos outros programas. Observa-se que existe um vazio de dados no intervalo de $40-50^{\circ}$ norte, mas ao avaliar os dados inseridos nota-se que desaparecera a atitude de N48W 40NE (atitude clar 42/40), que deveria estar inserida no intervalo. A plotagem dos dados é idêntica entre os softwares, conforme ilustrado na Figura 10, usando os dados da Tabela 5 .

Tabela 5. Medidas de atitudes de falhas. Fonte: Torquato et al. (1996, p.110)

\begin{tabular}{c|c}
\hline Planos de falhas & Estrias \\
\hline 1) N-S 90 & 1) $184 / 10$ \\
\hline 2) N20W 90 & 2) $160 / 62$ \\
\hline 3) N50W 90 & 3) $130 / 75$ \\
\hline 4) N60W 90 & 4) $120 / 78$ \\
\hline 5) N10W 90 & 5) $170 / 40$ \\
\hline 6) N40W 90 & 6) $140 / 73$ \\
\hline 7) E-W 90 & 7) $090 / 79$ \\
\hline 8) N27W 90 & 8) $154 / 63$ \\
\hline
\end{tabular}

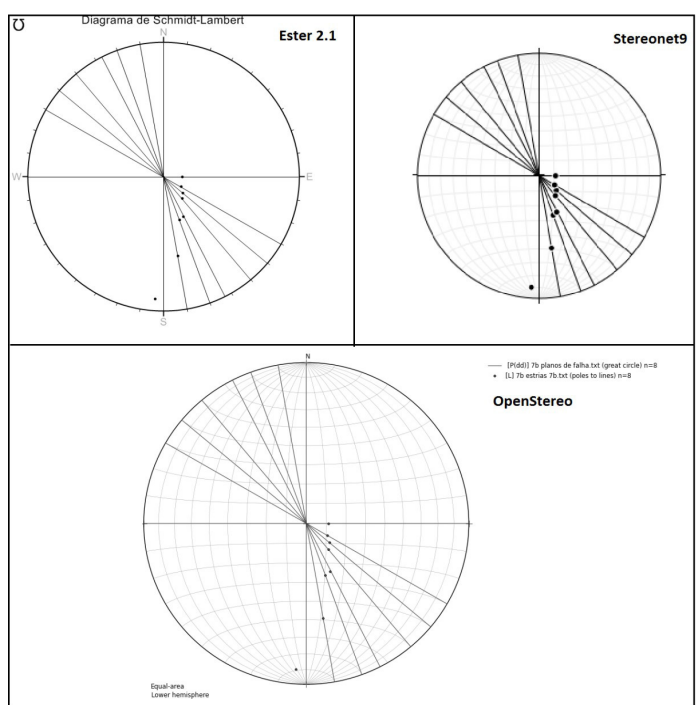

Figura 10. Resultados gerados pelos softwares, Ester 2.1, Stereonet9 e OpenStereo, com os dados da tabela 5

Utilizando os dados da Tabela 2, foi comparada a plotagem concomitante de dados de polos de estruturas planares e dados lineares. A plotagem mostra-se idêntica, existindo uma dificuldade de visualização no software Stereonet9; a distinção entre linhas e polos no programa OpenStereo pode ser realizada pela cor graças à necessidade de criar dois arquivos textos que são inseridos em separado. O protótipo Ester 2.1 oferece a opção de diferenciar as cores, mas os símbolos não podem ser alterados, constituindo default do programa. Os diagramas gerados estão presentes na Figura 11.
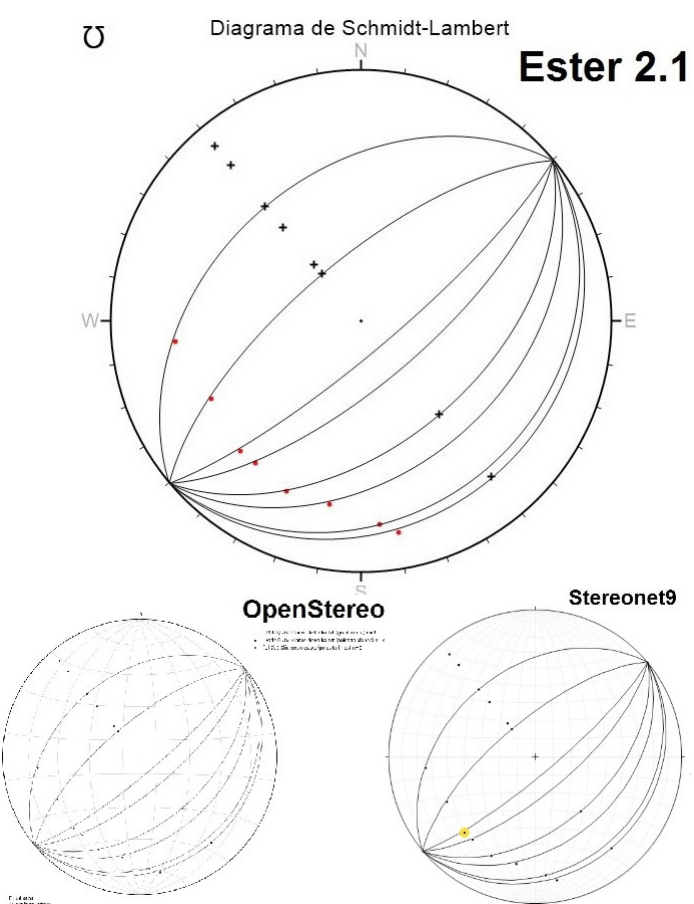

Figura 11. Resultados gerados pelos softwares, Ester 2.1, Stereonet9 e OpenStereo, com os dados da tabela 2

Para analisar os contornos gerados pelos softwares com relação à densidade de 'pontos', na representação gráfica de dados lineares, utilizou-se o conjunto de dados presentes na Tabela 3 , avaliando assim polos e lineações em um único conjunto. Os resultados gerados pelos programas estão dispostos na Figura 12. Apenas o programa Ester 2.1 consegue trabalhar com os dados de planos e linhas em uma única densidade, os demais softwares têm de elaborar um diagrama para cada tipo de dado.

Ester 2.1 permite mudar a cor dos dados individualmente; assim, foliações que possuem caimento para oeste foram plotadas em vermelho (Fig. 12), enquanto as que exibem mergulho para sul mantiveram a cor em preto. O software Stereonet 9 apresentou composição duplicada de diagramas em roseta, um para o mergulho das camadas e outro para os polos, sendo impossível apresentar apenas um desses e manter a ilustração dos polos e dos

\begin{tabular}{c|c|c|c|c|c}
\hline (c) Terrae Didat & Campinas, SP & v.14 & n.1 & p. $15-26$ & jan./mar. 2018 \\
\hline \multicolumn{2}{|c|}{23}
\end{tabular}


planos. Também não existe a opção de alterar as cores presentes. Já no programa OpenStereo não é possível colocar a roseta junto com o diagrama dos dados; o software OpenStereo apresenta, ainda, um distinto agrupamento de dados, com relação aos outros dois softwares.

\section{Conclusões}

Os testes de utilização do protótipo revelaram o bom funcionamento de Ester 2.1, tendo sido produzida uma série extensa de diagramas para avaliação comparativa com os produtos gerados em programas similares e disponíveis no mercado, como Stereonet9, OpenStereo e outros, que são acessíveis a baixo custo. O mais importante diferencial do programa Ester 2.1 em relação aos demais é gerar o diagrama tangente, cujas vantagens ainda não foram devidamente exploradas no meio técnico nacional. Tal como o diagrama Polar, a rede Tangente dispensa as operações de rotação para fazer plotagens manuais. Da mesma forma, torna desnecessário converter planos em polos e vice-versa antes das plotagens.

A operação de Ester 2.1 se diferencia na elaboração de diagramas de roseta apenas quando comparado com Stereonet 9 , mas é concordante com os demais softwares. Utilizando-se Ester 2.1 é possível gerar diagramas de densidade com dados de polos e lineações juntos, mas a resolução da imagem ainda é baixa e precisa ser melhorada. É grande a facilidade de se identificar linhas (pontos), planos e polos (pequenas cruzes) no ambiente Ester 2.1, com relação aos demais softwares, devido ao fato de que também é possível alterar a coloração individual de cada dado inserido. Não só os dados, mas também os gráficos gerados podem ter as cores alteradas, recursos que não estão presentes nos demais softwares. Associando todas as características, o software Ester 2.1 mostrou-se completo e sintético, possuindo interface bem amigável para usuários inexperientes em projeção estereográfica.

\section{Agradecimentos}

Os autores agradecem ao $\mathrm{CNPq}$, Conselho Nacional de Desenvolvimento Científico e Tecnológico, pela concessão de Bolsa de Produtividade em Pesquisa, nível 2, ao primeiro autor (CDRC), e ao Programa PIBIC-CNPq, pelas bolsas de iniciação científica que possibilitaram o amadurecimento acadêmico de vários estudantes do curso de graduação em Geologia, dentre os quais se incluem o segundo e o terceiro autores. Agradecem ainda aos professores Paulo Cesar Soares e Francisco A. Matos de Abreu pelas proveitosas sugestões que fizeram ao manuscrito.

\section{Referências}

Allmendinger R. 2017. Stereonet9. Structural Geology Algorithms for Windows v. 9.9.6 (64-bit). Version 9.9.6 - 2017.08.11. URL: http:/www.geo.cornell. edu/geology/faculty/RWA/programs/stereonet.html. Acesso 24.10.2017.

Aleksandrowski P. 1985. Graphical determination of principal stress directions for slickenside lineation population: an attempt to modify Arthaud's method. J. Struct. Geol., 7(1):73-82.

Bengtson C.A. 1980. Structural uses of tangent diagrams. Geology, 8:599-602.

Blenkinsop T. 1999. Pedagogy of stereographic projection. J. African Earth Sciences, 28(4):897-902. URL: http://www.sciencedirect.com/science/article/pii/ S0899536299000664. Acesso 3.04.2014.

Burger H.R., Bykerk-Kauffman A., Guth L.R., Hurst S.D., Moore A.M., Onasch C.M., Reynolds S.J. 2004. Effective use of computers in teaching struc-

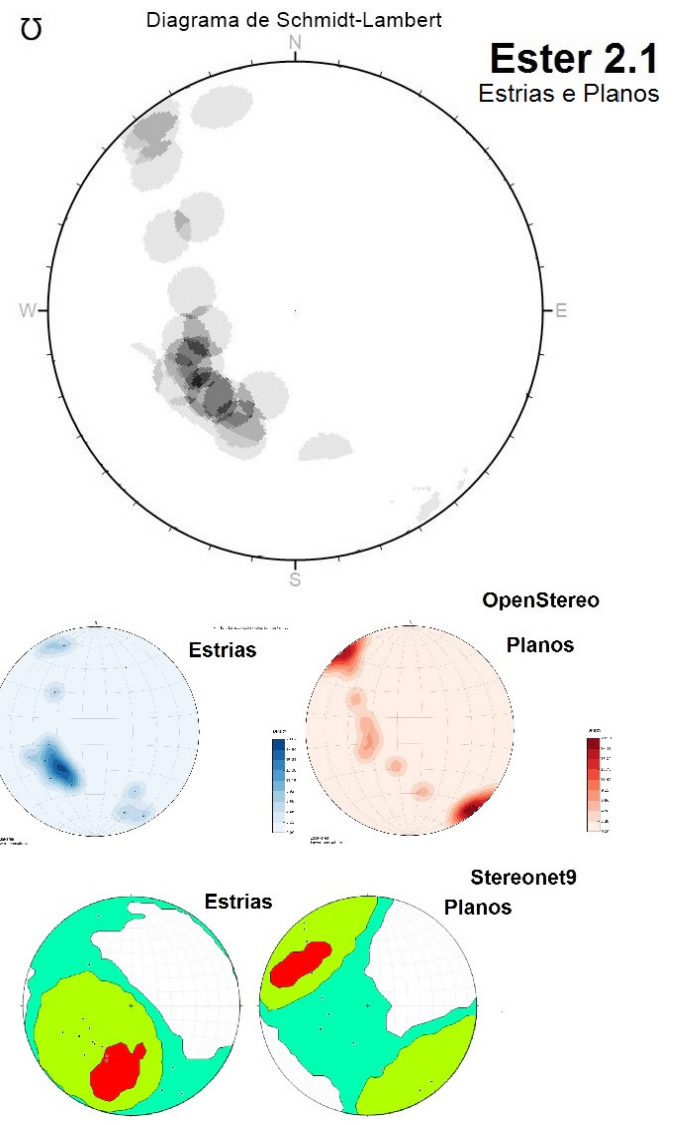

Figura 12. Resultados gerados pelos softwares Ester 2.1, Stereonet9 e OpenStereo, com os dados da Tabela 3 
tural geology. In: 2004 GSA Annual Meeting, Denver, CO, 2004. Special Session on Teaching Structural Geology... Denver, CO: Geological Society of America. URL: https://gsa.confex.com/gsa/2004AM/finalprogram/abstract_75901.htm. Acesso 26.04.2017.

Campanha G.A.C., Carneiro C.D.R., Pereira Jr. G.G., Furumoto S., Hasui Y., Nagata N. 1996. Uso do programa Trade para determinação de direções principais de esforços pelos métodos de Arthaud e Angelier. In: C.D.R. Carneiro (coord.) 1996. Projeção estereográfica para análise de estruturas. Programas ESTER e TRADE. Fundamentos Teóricos, Exercícios e Aplicações em Microcomputador, Laboratório e Campo. São Paulo: Co-Edição CPRM / IG-Unicamp / IPT-Digeo. p.115-120.

Carneiro C.D.R. coord. 1996. Projeção estereográfica para análise de estruturas. Programas ESTER e TRADE. Fundamentos Teóricos, Exercícios e Aplicações em Microcomputador, Laboratório e Campo. São Paulo: Co-Edição CPRM / IG-Unicamp / IPT-Digeo. $184 \mathrm{p}$.

Carneiro C.D.R., Carvalho A.M.A.de 2008. CAD resources for resolving Structural Geology problems. In: Intern. Geol. Congr., 33, Oslo, 2008. Abstract CD-ROM... Oslo: IUGS. (Symp. IEI-01 General contributions to geoscience information). URL: http://www.cprm.gov.br/33IGC/1344380.html. Acesso 26.11.2010.

Carneiro C.D.R., Carvalho A.M.A.de. 2012. Utilização de recursos de ambiente CAD em Geologia Estrutural. Terre Didatica, 8(2):83-93. DOI: 10.20396/ td.v8i2.8637417. URL: https://periodicos.sbu.unicamp.br/ojs/index.php/ td/article/view/8637417. Acesso 10.10.2017.

Frodeman R.L. 1995. Geological reasoning: Geology as an interpretive and historical science. Geol. Soc. Am. Bull., 107(8):960-968.

Frodeman R. 2010. O raciocínio geológico: a geologia como uma ciência interpretativa e histórica. Trad. L.M. Fantinel \& E.V.D. Santos. Terre Didatica, 6(2):85-99. DOI: 10.20396/td.v6i2.8637460. URL: https://periodicos.sbu.unicamp.br/ojs/index.php/td/ article/view/8637460. Acesso 23.10.2017.

Groshong Jr. R.H. 2006. 3-D Structural Geology: a practical guide to quantitative surface and subsurface map interpretation. New York, Springer, 400p. (CD-ROM incl.).

Grohmann C.H., Campanha G.A.C. 2010. OpenStereo: open source, cross-platform software for structural geology analysis. AGU Fall Meeting 2010, San Francisco, California, USA. Abstracts... AGU.

Grohmann C.H., Campanha G.A.C., Soares Jr. A.V. 2011. Openstereo: um programa livre e multiplataforma para análise de dados estruturais. In: Simp. Nac. Est. Tectônicos, 13, Campinas, 2011 e Intern. Simp. on Tectonics, 7, Campinas, 2011. Anais VIII SNET..., Campinas: SBG/NE. p. 26-28. (CDROM). URL: http://www.sp.sbgeo.org.br/SBG-SP/ Eventos_files/Anais_13_SNET_2011.pdf. Acesso 24.10.2017.

Guth P.L. 1987. MICRONET: interactive equal area and equal angle nets. Computers \& Geosciences, 13(5):541-543.

Holcombe R.J. 2003. GEOrient (C), v. 9.1. URL: http:// www.holcombe.net.au/software/georient.html.
Acesso 3.05.2017.

Hubbert M.K. 1931. Graphic solution of strike and dip from two angular components. Am. Assoc. Pet. Geol. Bull., 15(1):283-286.

Jacobson C.E. 2001a. Using AutoCAD for descriptive geometry exercises in undergraduate Structural Geology. Computers \& Geosciences, 27(1):9-15. doi $>10.1016 / S 0098-3004(00) 00060-1 . \quad$ Acesso 26.09.2017

Jacobson C.E. 2001b. Using AutoCAD for Descriptive Geometry Exercises in Undergraduate Structural Geology (Expanded version of manuscript published in Computers \& Geosciences, 27). doi >10.1016/S00983004(00)00060-1, URL: http://portal.acm.org/citation.cfm?id=372821. Acesso 26.09.2017.

James P.R., Clark I. 1996. GeologiCAl Structure. Multimedia Presentation and Modelling Software. In: DE PAOR, D.G. 1996. Structural Geology and personal computers. New York, Pergamon. p.13-37.

Kastens K., Manduca C.A., Cervato C., Frodeman R., Goodwin C., Liben L.S., Mogk D.W., Spangler T.C., Stillings N.A. and Titus S. 2009. How Geoscientists think and learn, Eos Trans. AGU, 90(31):265. URL: serc.carleton.edu/serc/EOS-90-31-2009.html. Acesso 26.04 .2017$.

Kitson H.W. 1929. Graphic solution of strike and dip from two angular components. Am. Assoc. Pet. Geol. Bull., 13(9):1211-1213.

Knox-Robinson C.M., Gardoll S.J. 1998. GIS-Stereoplot: an interactive stereonet plotting module for arcview 3.0 geographic information system. Computers \& Geosciences, 24(3):243-250.

Miguel, G.F., Carneiro C.D.R., Guimarães G.A., Souza J.P.P. 2017. A projeção estereográfica como recurso essencial para aprendizagem significativa em Geologia Estrutural. Rev. de la Enseñanza de las Ciencias de la Tierra.

Mizuno T.A., Carneiro C.D.R. 2007. Atualização de softwares de projeção estereográfica para sistema operacional 32 bits, a partir de Ester 1.0 - DOS. In: Congr. Interno Inic. Cient., 15, Campinas, 2007. Resumos... Campinas: Unicamp. [pdfN/995.pdf] (CDROM, resumo E0367)

Mizuno T.A., Carneiro C.D.R. 2008. Implementação e divulgação de software de projeção estereográfica em sistema operacional 32 bits. In: Congr. Interno Inic. Cient., 16, Campinas, 2008. Resumos... Campinas: Unicamp. p. 37. [pdfN/844.pdf] (CD-ROM, resumo E0446)

Mynatt I., Bergbauer S., Pollard D.D. 2007. Using differential geometry to describe 3-D folds. J. Struct. Geol., 29:1256-1266.

Nettleton L.L. 1931. Graphic solution of strike and dip from two angular components. Am. Assoc. Pet. Geol. Bull., 15(1):79-82.

Railsback B. 2002a. How geologists think. A quick introduction for earth-science students. Dept. Geol., Univ. Georgia. URL: http://www.gly.uga.edu/railsback/geointro/GeologyBackground1.html. Acesso 03.05.2017.

Railsback B. 2002b. What geologists see in a landscape. Dept. Geol., Univ. Georgia. URL: http://www.gly. uga.edu/railsback/geointro/GeologyBackground2. html. Acesso 03.05.2017.

Souza J.P.P., Carneiro C.D.R. 2013. Atualização de 
programa de projeção estereográfica em ambiente Windows e criação de materiais educacionais a partir dos programas ESTER e TRADE. In: Congr. Interno Inic. Cient., 21, Campinas, 2013. Resumos... Campinas: UNICAMP. [Resumo E0563] (CDROM, URL: https://www.prp.unicamp.br/pibic/ congressos/xxicongresso/cdrom/FSCOMMAND/ pdfN/563.pdf. Acesso 12.01.2018.

Stowe C.W. 1988. A pascal program for plotting and rotating stereographic projections. S. Afr. J. Geol., 91(4):527-542.

Torquato J.R., Pedreira L.H., Carneiro C.D.R., Hasui Y. 1996. Métodos gráficos para determinação das direções principais de esforços em áreas afetadas por falhas. In: C.D.R. Carneiro (coord.) 1996. Projeção estereográfica para análise de estruturas. Programas ESTER e TRADE. Fundamentos Teóricos, Exercícios e Aplicações em Microcomputador, Laboratório e Campo. São Paulo: Co-Edição CPRM / IG-Unicamp / IPT-Digeo. p.99-113.

Van Everdingen D., Van Gool, J. 1990. QuickPlot, plotting program for orientation data. Newfoundland, Dept. Earth Sci., Memorial Univ. of Newfoundland.

Van Everdingen D., Van Gool J., Vissers R.L.M. 1992. Quickplot: a microcomputer-based program for processing of orientation data. Computers \& Geosciences, 18(2/3):183-287. DOI: https:// doi.org/10.1016/0098-3004(92)90081-2. Acesso 26.04.2017. 